Honam Mathematical J. 35 (2013), No. 3, pp. 389-394

http://dx.doi.org/10.5831/HMJ.2013.35.3.389

\title{
FIXED POINTS AND KERNEL OF THE PROJECTIVE HOLONOMY OF AN AFFINE MANIFOLD
}

\author{
KYEONGSU PARK
}

\begin{abstract}
It is an interesting problem to study fixed points of an element in the holonomy group of an affine manifold. We compute the limit of a sequence of projective transformations and verify relations between fixed points and kernels.
\end{abstract}

\section{Introduction}

An affine manifold $M$ is a smooth manifold whose coordinate transitions are affine maps. Since affine maps are analytic, an affine manifold is an analytic manifold. See [1], [2] and [6].

A coordinate chart can be analytically continued along a curve and induces a map $D$ from the universal covering space $\tilde{M}$ to $\mathbb{R}^{n}$. Obviously, $D$ depends on the choice of the initial chart. The map $D$ is called a developing map and the image $D(\tilde{M})$ is called a developing image.

For a deck transformation $\alpha \in \pi_{1}(M)$ there is an affine transformation $\rho(\alpha)$ such that

$$
D(\alpha x)=\rho(\alpha) D(x) .
$$

The map $\rho$ is a homomorphism from $\pi_{1}(M)$ into the affine group $\operatorname{Aff}(n)$ of $\mathbb{R}^{n}$. $\rho$ is called the holonomy homomorphism and $\Gamma=\rho\left(\pi_{1}(M)\right)$ the holonomy group.

Now we especially consider the homomorphism $\psi$ from $\operatorname{Aff}(n)$ to $\operatorname{PGL}(n+1, \mathbb{R})$ given by the composition of the following two homomorphisms:

$$
\begin{aligned}
& \operatorname{Aff}(n) \quad \longrightarrow \quad \mathrm{GL}(n+1, \mathbb{R}) \quad \longrightarrow \quad \operatorname{PGL}(n+1, \mathbb{R}) \\
& (a, A) \longmapsto\left(\begin{array}{cc}
A & a \\
0 & 1
\end{array}\right) \longmapsto \quad\left[\begin{array}{cc}
A & a \\
0 & 1
\end{array}\right],
\end{aligned}
$$

Received May 3, 2013. Accepted June 26, 2013.

2010 Mathematics Subject Classification. 57N10, 57N15.

Key words and phrases. affine manifold, projective holonomy. 
where $\left[\begin{array}{cc}A & a \\ 0 & 1\end{array}\right]$ is the equivalence class containing $\left(\begin{array}{cc}A & a \\ 0 & 1\end{array}\right)$. The group $\psi(\Gamma)$ is called the projective holonomy group of the affine manifold $M$.

In this paper, we show that there is a singular projective transformation $\sigma \in \overline{\psi(\Gamma)}$ such that $[\operatorname{ker} \sigma] \cap \mathbb{R}^{n} \neq \emptyset$ if $M$ is closed and an element of $\Gamma$ has a fixed point in $\mathbb{R}^{n}$.

T. Nagano and K. Yagi proved a result related to fixed points of holonomy group ([4]): If the holonomy group $\Gamma$ of a closed affine manifold has a fixed point then the developing image avoids the fixed point.

\section{Limit of a sequence of projective transformations}

In this section we describe limits of sequences of projective transformations.

Let $\mathrm{M}(n)$ be the set of $n \times n$ matrices whose entries are real numbers. $\mathrm{M}(n)$ can be identified with $\mathbb{R}^{n^{2}}$ and therefore, projectivized. The projectivization of $\mathrm{M}(n)$ is denoted by $\operatorname{PM}(n)$. The equivalence class $[L] \in \operatorname{PM}(n)$ containing $L$ is called the projectivization of $L$. That is,

$$
[L]=\{X \in \mathrm{M}(n)-\{O\} \mid X=k L \text { for some } k \in \mathbb{R}-\{0\}\} .
$$

The equivalence class $[L]$ can be considered as a map from $\mathbb{R} \mathrm{P}^{n-1}$ onto itself whenever $L$ is not singular. It is called a nonsingular projective transformation. The projective general linear group $\operatorname{PGL}(n)$, or $\operatorname{PGL}(n, \mathbb{R})$, is the group of non-singular projective transformations.

For a singular matrix $L$ the projectivization of $\operatorname{ker} L$ is a projective subspace of $\mathbb{R P}^{n-1}$. We call it the projective kernel of $[L]$ and denote $\operatorname{ker}[L]$. The equivalence class $[L]$ can be considered as a map from $\mathbb{R} \mathrm{P}^{n-1}-\operatorname{ker}[L]$ to $\mathbb{R} \mathrm{P}^{n-1}$. It is called a singular projective transformation. See also [3].

Let $L$ be an $n \times n$ matrix with real entries and $\lambda_{1}, \ldots, \lambda_{s}$ complex eigenvalues of $L$. There exist square matrices $\Lambda_{1}, \ldots, \Lambda_{s}$ such that

$$
\Lambda_{r}=\left(\begin{array}{ccc}
\lambda_{r} & 1 & \\
& \ddots & 1 \\
& & \lambda_{r}
\end{array}\right) \text {, }
$$

and $L$ is similar to

$$
\left(\begin{array}{lll}
\Lambda_{1} & & \\
& \ddots & \\
& & \Lambda_{s}
\end{array}\right)
$$


When $\lambda_{r}=a_{r}+i b_{r}$ is not a real number, $\bar{\lambda}_{r}$ also is an eigenvalue and one of $\Lambda_{q}$ coincides with

$$
\bar{\Lambda}_{r}=\left(\begin{array}{ccc}
\bar{\lambda}_{r} & 1 & \\
& \ddots & 1 \\
& & \bar{\lambda}_{r}
\end{array}\right) .
$$

We note that $\left(\begin{array}{cc}\Lambda_{r} & 0 \\ 0 & \bar{\Lambda}_{r}\end{array}\right)$ is similar to

$$
\left(\begin{array}{ccc}
C_{r} & I & \\
& \ddots & I \\
& & C_{r}
\end{array}\right)
$$

where $C_{r}=\left(\begin{array}{cc}a_{r} & -b_{r} \\ b_{r} & a_{r}\end{array}\right)$. Hence $L$ is similar to

$$
\left(\begin{array}{lll}
A_{1} & & \\
& \ddots & \\
& & A_{l}
\end{array}\right)
$$

where $A_{k}$ is of the form (1) with a real number $\lambda_{r}$ or (2) with a complex number $\lambda_{r}=a_{r}+i b_{r}$.

Suppose that $\Lambda \in \mathrm{M}(n)$ is a matrix of the form (1) with a real number $\lambda=\lambda_{r}$. For sufficiently large $m$ we obtain

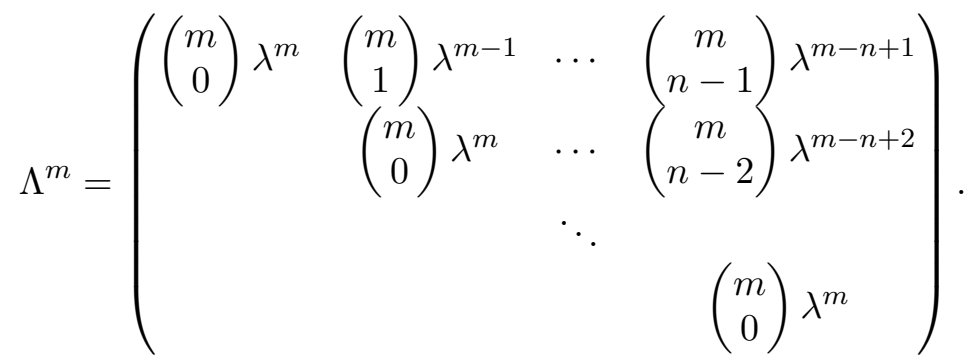

The $(1, n)$-entry $\left(\begin{array}{c}m \\ n-1\end{array}\right) \lambda^{m-n+1}$ has the largest absolute value among entries of $\Lambda^{m}$. Hence $[\Lambda]^{m}$ converges to a projective transformation $\left[\Lambda_{0}\right]$ and $\Lambda_{0}$ has only a nonzero entry at $(1, n)$. The matrix $\Lambda_{0}$ is not singular if and only if $n=1$ and $\lambda \neq 0$. The kernel $\Lambda_{0}$ is of dimension $n-1$ if $\lambda \neq 0$.

Suppose that $\Lambda \in \mathrm{M}(n)$ is a matrix of the form (2) with a complex number $\lambda_{r}$. The similar arguments implies that the sequence $[\Lambda]^{m}$ has a convergent subsequence. The limit $\left[\Lambda_{0}\right]$ of the subsequence is a singular 
projective transformation if and only if $n>2$. The kernel $\operatorname{ker} \Lambda_{0}$ is of dimension $n-2$.

In general, suppose that $L$ is of the form (3). Let $\left[L_{0}\right]$ be the limit of a convergent subsequence of $[L]^{m}$ and

$$
\mu=\max \left\{\left|\lambda_{1}\right|, \ldots,\left|\lambda_{s}\right|\right\} .
$$

If $\left|\lambda_{r}\right|<\mu$ for some $r$ and the submatrix $A_{k}$ is of the form (1) with a real number $\lambda_{r}$ or (2) with a complex number $\lambda_{r}$ then $L_{0}$ is singular.

If $\left|\lambda_{r}\right|=\mu$ for all $r$ and $L$ is diagonalizable then $L_{0}$ is nonsingular. As a result,

Proposition 1. Suppose that $L \in \mathrm{M}(n)$ is a non-singular matrix and a subsequence of $\left[L^{m}\right]$ converges to $\left[L_{0}\right] . L_{0}$ is non-singular if and only if $L$ is similar to $c R$ for a nonzero real number $c$ and an orthogonal matrix $R$.

Proof. We note that a matrix is orthogonal if and only if it is similar to (3) where $A_{k}$ is one of the following 4 forms:

$$
(1),(-1),\left(\begin{array}{cc}
\cos \theta & -\sin \theta \\
\sin \theta & \cos \theta
\end{array}\right),\left(\begin{array}{cc}
-\cos \theta & \sin \theta \\
\sin \theta & \cos \theta
\end{array}\right) .
$$

Applying the above arguments, our result follows.

\section{Fixed point of an affine holonomy}

Suppose that an affine transformation $(a, A)$ has a fixed point, That is, there exists a point $x \in \mathbb{R}^{n}$ such that $a+A x=x$. Let

$$
L=\left(\begin{array}{cc}
A & a \\
0 & 1
\end{array}\right), \quad \xi=\left(\begin{array}{l}
x \\
1
\end{array}\right) .
$$

Then the nonsingular matrix $L$ has a point $\xi$ as a fixed point. Hence $\xi$ is an eigenvector of $L$ with the corresponding eigenvalue 1 .

Suppose that $L$ has an eigenvalue $\lambda$ such that $|\lambda|>1$. The arguments in section 2 implies that $[L]^{m}$ has a subsequence which converges to $\left[L_{0}\right]$ and $\xi \in \operatorname{ker} L_{0}$.

Suppose that $L$ has an eigenvalue $\lambda$ and $|\lambda|<1$. Then $\xi$ is a fixed point of

$$
L^{-1}=\left(\begin{array}{cc}
A^{-1} & -A^{-1} a \\
0 & 1
\end{array}\right)
$$

and $L^{-1}$ has an eigenvalue $1 / \lambda$ and $|1 / \lambda|>1$.

If $L$ is not similar to an orthogonal matrix and any eigenvalue of $L$ is of absolute value 1 , then $[L]^{m}$ has a subsequence which converges to 
$\left[L_{0}\right]$ and $\xi \in \operatorname{ker} L_{0}$ as section 2 . Hence we have

Theorem 1. Let $M$ be an affine manifold with the holonomy group $\Gamma$. If $\gamma \in \Gamma$ has a fixed point and is not similar to an orthogonal matrix, then there is a singular projective transformation $\sigma \in \overline{\psi(\Gamma)}$ such that $\operatorname{ker} \sigma \cap \mathbb{R}^{n} \neq \emptyset$.

Now we introduce a set, which is called a limit set, defined in [5]. Let $M$ be an affine manifold with the developing map $D: \tilde{M} \rightarrow \mathbb{R}^{n}$. Let $E_{M}$ be the set of those points $y$ which is the end point $c(1)$ of a continuous curve $c$ in $\mathbb{R}^{n}$ such that :

(4) there exists a curve $\tilde{c}(t) \in \tilde{M}$ for $0 \leq t<1, D(\tilde{c}(t))=c(t)$ and $\tilde{c}(1)$ can not be defined continuously in $\tilde{M}$.

The following Theorem is proved in [5].

Theorem 2. Let $M$ be a closed affine manifold. For any $x \in E_{M}$ there exists $\sigma \in \overline{\psi(\Gamma)}$ such that $x \in \operatorname{ker} \sigma$.

Now, suppose that $M$ is a closed affine manifold and $(a, A) \in \Gamma$ has a fixed point $x$. If $x \notin \Omega$ then obviously $E_{M}$ is not empty.

We assume that $x=D(u) \in \Omega$ for some $u \in \tilde{M}$. Let $\alpha: \tilde{M} \rightarrow \tilde{M}$ be a deck transformation satisfying $\rho(\alpha)=(a, A)$ and $h:[0,1] \rightarrow \tilde{M}$ a curve starting at $u$ ending at $\alpha(u)$. The curve $D \circ h$ is a loop such that $(D \circ h)(0)=(D \circ h)(1)=x$. Hence there is an homotopy

$$
H:[0,1] \times[0,1] \rightarrow \mathbb{R}^{n}
$$

satisfying

$$
H(\cdot, 0)=D \circ h
$$

and

$$
H(\cdot, 1)=H(0, \cdot)=H(1, \cdot)=x .
$$

Since $u \neq \alpha(u)$, there is no homotopy

$$
\tilde{H}:[0,1] \times[0,1] \rightarrow \tilde{M}
$$

such that $D \circ \tilde{H}=H$. Hence a curve $c=H\left(t_{0}, \cdot\right)$ starting at $(D \circ h)\left(t_{0}\right)$ for some $t_{0}$ satisfies the condition (4).

In fact, $\tilde{c}$ in (4) with $D \circ \tilde{c}=c$ is not defined on $[0,1)$ but on $\left[0, t_{1}\right)$ for some $t_{1} \in(0,1)$ in this case.

Theorem 3. Let $M$ be a closed affine manifold. If an element in the holonomy group $\Gamma$ has a fixed point, then there is a singular projective 
transformation $\sigma \in \overline{\psi(\Gamma)}$ such that $\operatorname{ker} \sigma \cap \mathbb{R}^{n} \neq \emptyset$.

Proof. Since $E_{M}$ is not empty, Theorem 3 implies our result.

\section{References}

[1] Ch. Ehresmann, Sur les espaces localement homogenes, Enseign. Math. 35 (1936), 317-333.

[2] R. S. Kulkarni, On The Principle of Uniformization, Jour. Diff. Geom. 13 (1978), 109-138.

[3] P. J. Myberg, Untersuchungen über die automorphen Funktionen beliebig vieler Variablen, Acta Math. 46 (1925), 215-336.

[4] T. Nagano and K. Yagi, The Affine Structures On The Real Two-Torus (I), Osaka J. Math., 11 (1974), 181-210.

[5] K. Park Limit Sets of Projectively Flat Manifolds, Comm. Korean Math. Soc. 15(3) (2000), 541-547.

[6] D. Sullivan and W. Thurston, Manifolds with Canonical Coordinate Charts: Some Examples, Endeign. Math. 29 (1983), 15-25.

Kyeongsu Park

Department of Game, Jeonju University,

Jeonju 560-759, Korea.

E-mail: pine@jj.ac.kr 